\title{
Predictive Control Allocation for a Thermal Management System Based on an Inner Loop Reference Model—Design, Analysis, and Experimental Results
}

\author{
Chris Vermillion, Member, IEEE, Jing Sun, Fellow, IEEE, and Ken Butts, Member, IEEE
}

\begin{abstract}
This paper addresses the challenge of controlling an overactuated engine thermal management system where two actuators, with different dynamic authorities and saturation limits, are used to obtain tight temperature regulation. A modular control strategy is proposed that combines model predictive control allocation (MPCA) with the use of an inner loop reference model. This results in an inner loop controller that closely matches a dynamic specification for input-output performance while addressing actuator dynamics and saturation constraints. This paper presents the design and implementation strategy and illustrates the effectiveness of the proposed solution through real-time simulation and experimental results.
\end{abstract}

Index Terms-Automotive control, decentralized control, model predictive control (MPC), powertrain control.

\section{INTRODUCTION AND PROBLEM FORMULATION}

M ANY applications involve overactuated systems, in which the number of control inputs exceeds the number of outputs that are being controlled to a setpoint. This setup provides control engineers with additional opportunities that would not otherwise be present in a "square" system (one with the same number of inputs as outputs). However, it is often the case that other performance considerations, such as transient performance and system efficiency, motivate, justify, and sometimes even mandate overactuation. Hence, the optimal control of overactuated systems represents an important topic. In particular, it is of great interest to consider control strategies that optimize performance in the presence of overactuation and constraints, and can be implemented with available computational resources.

This paper considers an overactuated engine thermal management system. The system, shown in Figs. 1 and 2, is used

Manuscript received June 23, 2009; revised March 09, 2010; accepted May 26, 2010. Manuscript received in final form June 12, 2010. Date of publication July 15, 2010; date of current version June 17, 2011. Recommended by Associate Editor R. Rajamani. This work was supported by Toyota Engineering and Manufacturing, North America.

C. Vermillion and K. Butts are with Toyota Technical Center, Ann Arbor, MI 48105 USA (e-mail: chris.vermillion@tema.toyota.com; ken.butts@tema. toyota.com).

J. Sun is with the Naval Architecture and Marine Engineering Department and the Electrical Engineering and Computer Science Department, University of Michigan, Ann Arbor, MI 48109 USA (e-mail: jingsun@ umich.edu).

Color versions of one or more of the figures in this paper are available online at http://ieeexplore.ieee.org.

Digital Object Identifier 10.1109/TCST.2010.2053370 in engine testing in order to provide tight control of oil temperature at the engine outlet (a similar system to that of Fig. 1 exists for engine coolant; this paper restricts its study to the oil system). The system consists of a heater, heat exchanger, and mixing valve, which are all housed in a compact cabinet that is connected via flexible piping to the engine block. Flow is generated through the engine oil pump, which delivers a flow rate that is proportional to engine speed (hence, flow rate cannot be adjusted freely over the course of an experiment). While the system is designed to control a single temperature (engine outlet temperature), its two actuators (the mixing valve and heater) both serve important purposes. The heater is needed to provide auxiliary heating at low speeds and loads, but acts as a slow source of actuation compared to the mixing valve, which can deliver a temperature change to the engine inlet very quickly. Both actuators possess hard saturation limits, and actuator saturation constraints are often active during operation. Given these characteristics, it is desirable to pursue a control strategy that coordinates the two control inputs in order to optimize performance while considering the dynamic authorities and saturation limits of the actuators. One key feature that is common to most overactuated systems, including the thermal management system studied here, is the presence of a signal that characterizes the overall effect of many actuators. This signal acts as a "virtual control" to the plant dynamics. For a multi-input, single-output (MISO) system, the following representation can be used to decompose the system into two subsystems after introducing the virtual control input

$$
\begin{aligned}
x_{1}(k+1) & =f_{1}\left(x_{1}(k), v(k), d(k)\right) \\
y(k) & =h\left(x_{1}(k)\right) \\
x_{2}(k+1) & =f_{2}\left(x_{1}^{\prime}(k), x_{2}(k), u(k), d(k)\right) \\
v(k) & =g\left(x_{2}(k)\right)
\end{aligned}
$$

where $u \in \mathbb{R}^{q}, d \in \mathbb{R}^{p}, y \in \mathbb{R}$, and $v \in \mathbb{R}$ represent the control inputs, the disturbance inputs, the performance output, and the virtual control input, respectively. $x_{1} \in \mathbb{R}^{n_{1}}$ represents the plant states, which are driven by the virtual control input, $v$, whereas $x_{2} \in \mathbb{R}^{n_{2}}$ represents the actuator states, which are driven by the real control inputs, $u . x_{1}^{\prime} \in \mathbb{R}^{n_{1}^{\prime}}$, with $n_{1}^{\prime} \leq n_{1}$, represents the subset of plant states that affect the actuator dynamics. The real control inputs, $u$, affect the plant states only through the virtual control input, $v$. The thermal management system can be cast in the form of (1) by taking the virtual control input $(v)$ to be the 


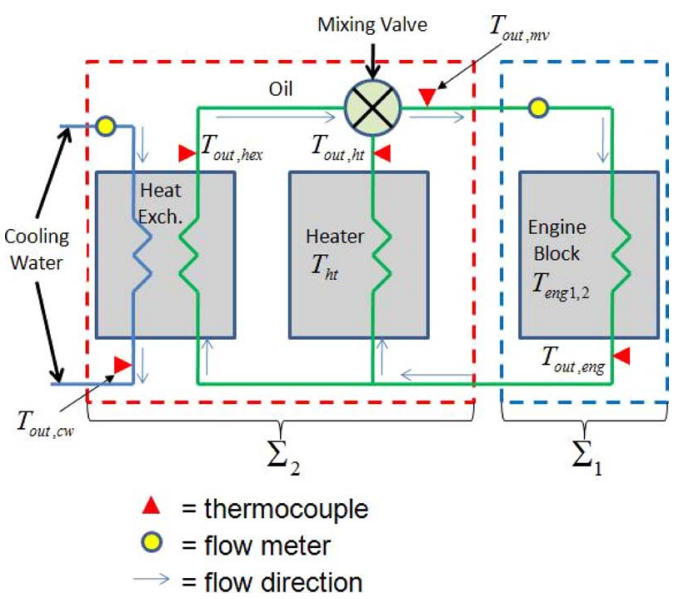

Fig. 1. Thermal management system diagram for the oil circuit, which will be the primary circuit under consideration in this paper. The layout of the coolant circuit is identical to that of the oil, although the various components are sized differently for engineering purposes.

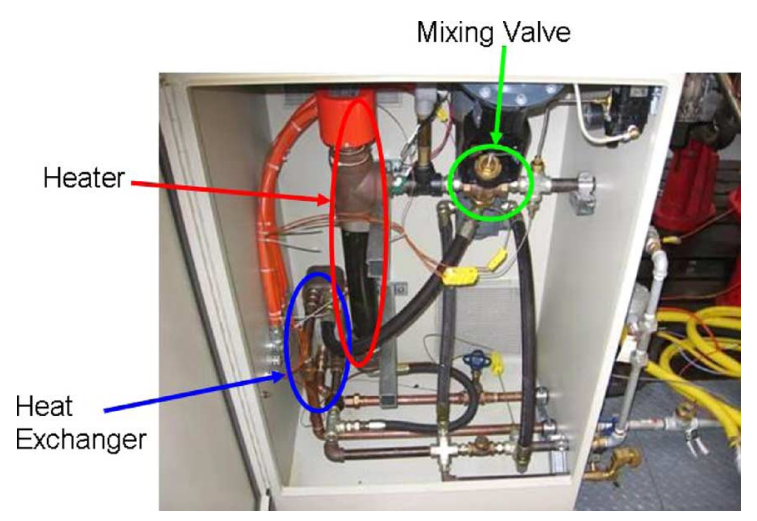

Fig. 2. Heater, heat exchanger, and mixing valve, which are housed in a unit separate from the engine.

mixing valve outlet temperature, where the performance output $(y)$ is the engine outlet temperature. For the thermal management system, disturbances come in the form of heat rejection through the engine block and variations of the temperature of the cooling water that is fed through the heat exchanger.

Remark 1.1: The primary topic of interest in this work will be setpoint tracking, with these disturbance values held constant and therefore taken as parameters. However, because many important test cell thermal management applications require the temperature to be held constant in the presence of engine load changes, this disturbance case will be treated in the Appendix.

The introduction of the virtual control input enables control designers to pursue a modular control strategy, as depicted in Fig. 3 (where the objective is to have $y$ track a setpoint, $r$ ), which divides one large control design task into two less complex design tasks. Here, an outer loop controller determines a desired virtual control input, $v_{\mathrm{des}}$, and an inner loop control allocation determines the real control inputs, $u$, that will be applied to the actuators in order to achieve close tracking of $v_{\text {des }}$. Since $v \in \mathbb{R}$ and there are no constraints on the virtual control input, the outer loop controller is single-input, single-output (SISO), and its design need not consider input constraints. On the other hand, the inner loop control allocation considers

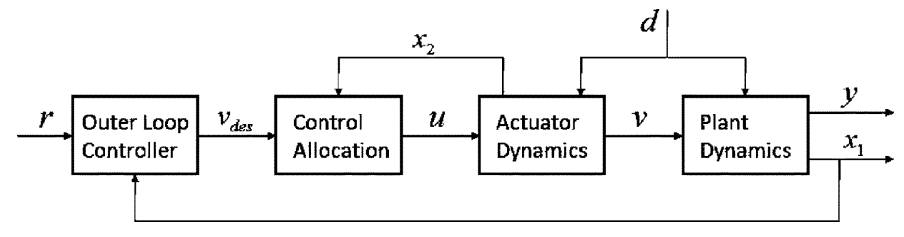

Fig. 3. Block diagram of the overall system under a modular control strategy.

both overactuation and actuator saturation constraints. It is at the inner loop control allocation level where the optimization of control inputs takes place. Thus, by pursuing a modular control strategy, the optimization of control inputs only needs to consider a subset of the full set of system states, thereby simplifying the optimization from what would be required under a centralized control strategy. For the thermal management system, the virtual control input is selected as the oil temperature at the outlet of the mixing valve. This virtual control input is conveniently located at the junction between the fluid conditioning system and the engine block, making the modular control strategy extremely attractive from a practical perspective. Given the non-negligible actuator dynamics in the thermal management system, this paper considers a dynamic control allocation problem (as opposed to earlier static control allocation problems [1]-[5]), as addressed in [6]-[10]. [7]-[10] employ a particularly attractive dynamic control allocation strategy, known as model predictive control allocation (MPCA). MPCA optimizes control inputs over a receding horizon in order to track the desired virtual control input closely while simultaneously considering actuator saturation constraints and actuator dynamics.

In the literature, various methods have been employed in an attempt to seamlessly integrate the outer and inner loops, including performing an inner loop "target state" calculation as in [6] and [8], [9], and employing a multi-rate control strategy [11] where the inner loop is updated at a faster rate than the outer loop. In spite of their different approaches, these methods are unified in their attempt to obtain tracking of $v_{\text {des }}$. Practicality of both the target calculation or multi-rate approach is contingent on sufficient time scale separation between the inner and outer loop dynamics, which often renders dynamic control allocation unnecessary in the first place.

This work proposes an alternative modular design and integration approach, which relies on an inner loop reference model that captures the desired inner loop input-output performance. An appropriately designed reference model describes inner loop input-output behavior that is achievable in the absence of saturation constraints, but may not be achievable when these constraints are active. The reference model-based inner loop design was used successfully in [12], where a closed form inner loop controller was employed. This work expands significantly upon the results from [12], combining the concepts of reference model-based control design with MPCA, which allows one to explicitly consider constraints in order to track the output of the reference model closely when constraints are active. Simulation and experimental results on the thermal management system will show the specific benefit of incorporating a carefully designed reference model. 
TABLE I

MODEL VARIABLES

\begin{tabular}{|c|c|c|}
\hline Generic Variable & Thermal Management Variable & Description \\
\hline$u$ & $\phi$ & Mixing valve position \\
& $\dot{Q}_{h t}$ & Heater power (kW) \\
& $\dot{Q}_{1}$ & \\
$\dot{Q}_{2}$ & Engine heat rejection in the vicinity of coolant (kW) \\
& $T_{\text {in }, c w}$ & Engine heat rejection in the vicinity of oil (kW) \\
& $T_{\text {out }, e n g}$ & Cooling water inlet temperature (C) \\
$x_{1}$ & $T_{\text {eng }}$ & Engine outlet temperature (C) \\
& $T_{\text {out }, m v}$ & Eng. block temp. in the vicinity of oil (C) \\
& $T_{\text {out }, h t}$ & Eng. block temp. in the vicinity of coolant (C) \\
$T_{h t}$ & Mixing valve outlet temperature (C) \\
& $T_{\text {out }, h e x}$ & Heater outlet temperature (C) \\
& $T_{\text {out }, c w}$ & Heater coil temperature (C) \\
& $T_{\text {out }, m v}$ & Heat exchanger outlet temperature (C) \\
& $T_{\text {out }, e n g}$ & Cooling water outlet temperature (C) \\
$y$ & & Mixing Valve Outlet Temperature (C) \\
& & Engine Outlet Temperature (C) \\
\hline
\end{tabular}

This paper is organized as follows. Section II describes the essentials of the thermal management system along with an analysis of important system characteristics. Section III provides a detailed description of the modular design framework and the proposed MPCA structure. Section IV shows the application of the design process on the thermal management system, and Sections V and VI present real-time simulation and experimental results, respectively. Conclusions and future work are given in Section VII.

\section{Thermal Management System Model Overview}

The MPCA framework investigated in this paper requires a control-oriented model of the thermal management system that includes both plant and actuator dynamics. The analysis of this model provides further justification for pursuing MPCA with a modular control strategy. Readers are referred to [13] and [14], which present the detailed model development that preceded any control design, as well as information on the legacy control strategy that was used in the test cell before this work. This section summarizes the model structure, with the specific equations provided in the Appendix. For model parameters, readers are referred to [14].

In the thermal management system model, the plant and actuator states, $x_{1,2}$, consist of temperatures, whereas the control inputs $(u)$ consist of the mixing valve position and the power supplied to the heater. Table I provides a list of all of the states and inputs for the thermal management system, noting the relationship between specific thermal management variables and their "generic equivalents" that correspond to the variables in Fig. 3. To be precise, mixing valve "position" refers to the ratio of the flow rate through the heater to the flow rate through the engine. The model is developed using thermodynamic first principles that can be found in [15], with the following simplifying assumptions:

A1) both coolant and oil have constant density and specific heat;
A2) heaters and heat exchangers assume lumped parameters, with no energy storage in the heat exchanger "core;"

A3) heater model assumes a single temperature state for the heater coil, allowing for energy storage in the coil.

A4) engine model assumes two states for engine block temperature, reflecting different temperature distribution and heat rejection dynamics across the engine block;

A5) mixing valve assumes adiabatic mixing;

A6) heat losses in pipes are neglected.

All of these assumptions are standard for similar control-oriented thermal system modeling. Additionally, model validation has shown these assumptions to be valid for the particular system studied in this work. Furthermore, analysis results in [13] have shown that each circuit (coolant and oil) is reasonably decoupled from the other, and control design and analysis can be performed for one circuit by holding the engine outlet temperature for the other circuit to a constant value that is representative of normal operation. For the simulation and experimental results in this work (with the exception of the Appendix), setpoint tracking at constant speed, load, and cooling water temperature will be considered, which implies that $T_{\mathrm{in}, \mathrm{cw}}$ and $\dot{Q}_{1,2}$ are constant.

Fig. 4 shows how each of the components of the system (heat exchanger, heater, valve, and engine block) interacts with the others.

\section{Reference Model ApProAch For Model Predictive CONTROL ALLOCATION}

This section describes the reference model based approach for MPCA, including the design framework and detailed MPCA formalism.

\section{A. Modular System Representation With an Inner Loop Reference Model}

The reference model based approach relies on the use of an inner loop reference model, which represents a design target for the inner loop control designer and a design assumption for 


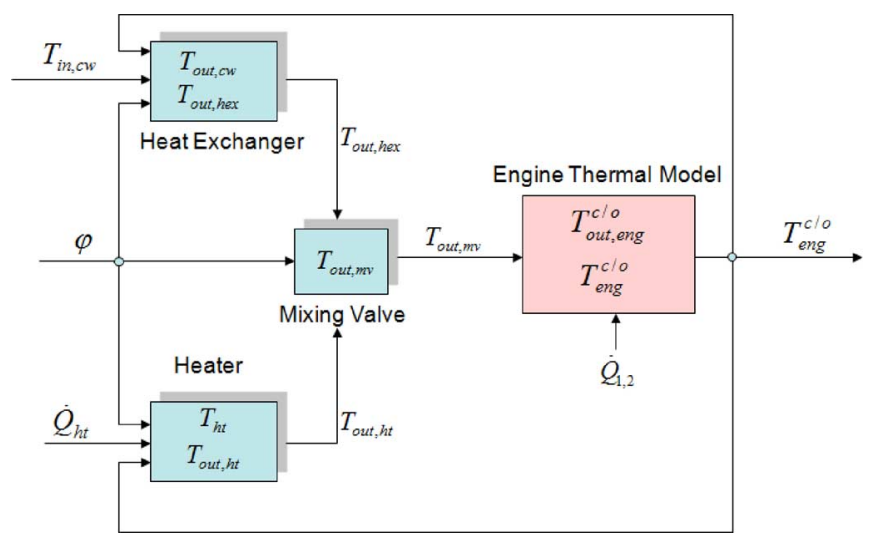

Fig. 4. Diagram of the signal flow between all of the interconnected subsystems in the engine thermal management system. Note that the flow rates through the heat exchanger and heater are functions of the valve position, $\phi$; hence, $\phi$ appears as an input to both of these subsystems.

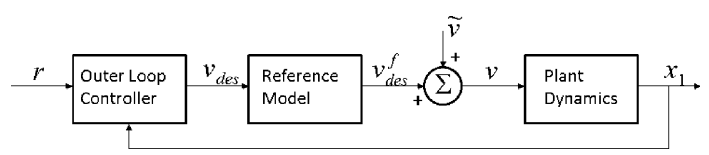

Fig. 5. Diagram illustrating the outer loop control design framework.

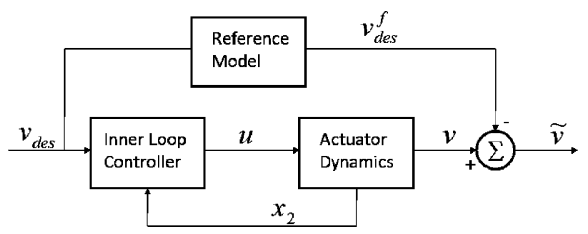

Fig. 6. Diagram illustrating the inner loop control design framework.

the outer loop control designer. With the reference model-based approach, the control design is carried out as follows:

1) define a reference model that represents a desirable and realistic performance target for the inner closed loop;

2) design the outer loop controller as in Fig. 5 with the assumption that the inner loop behavior matches that which is specified by the reference model;

3) for the inner loop control design, depicted in Fig. 6, instead of focusing on driving $v$ to $v_{\text {des }}$, design the inner closed loop to minimize the error between $v$ and the output of the reference model (with input $v_{\text {des }}$ ), which will be referred to as $v_{\text {des }}^{f}$.

While the first step is carried out prior to either the inner and outer loop design, the second and third steps can be carried out in parallel, by different designers. As one can see from Fig. 5, the outer loop controller can be designed to achieve a combined objective, consisting of tracking of the setpoint, $r$, and rejection of the disturbance seen by $\tilde{v}$, where $\tilde{v} \triangleq v-v_{\text {des }}^{f}$ (i.e., the error between the virtual control and the output of the reference model). As in Fig. 6, the inner loop control design can be cast as a reference model matching design, where the consequence is that $\tilde{v}$ asymptotically approaches zero. Without saturation constraints and with appropriate assumptions on the actuator dynamics and inner loop reference model, the well-known model reference control approach, shown for example in [16], can be used to design a closed-form inner loop controller that achieves this inner

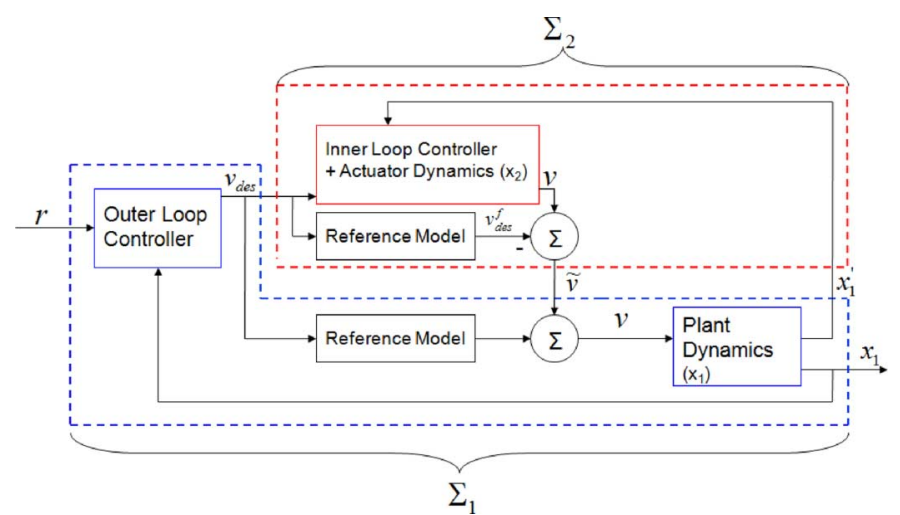

Fig. 7. Diagram of the system (1) recast for the reference model based design framework. The figure also reflects the distribution of design tasks (separated by dashed lines) between the inner and outer loop designs.

loop control objective. The objective of MPCA is to achieve this objective closely in the presence of constraints, whereas closedform model reference control designs do not provide such guarantees. To integrate the inner and outer loop controllers developed by the reference model based design process, the following non-minimal representation of the closed loop system is introduced, which is depicted in Fig. 7:

$$
\begin{aligned}
& \Sigma_{1}:=\left\{\begin{array}{l}
x_{1}^{c}(k+1)=f_{1}\left(x_{1}^{c}(k), v_{\mathrm{des}}^{f}(k), \tilde{v}(k)\right) \\
v_{\mathrm{des}}(k)=c_{1}\left(x_{1}^{c}(k), r(k)\right) \\
x_{1}^{\prime}(k)=g_{d}\left(x_{1}(k)\right) \\
x_{f}(k+1)=f_{3}\left(x_{f}(k), v_{\mathrm{des}}(k)\right) \\
v_{\mathrm{des}}^{f}(k)=g_{f}\left(x_{f}(k)\right)
\end{array}\right. \\
& \Sigma_{2}:=\left\{\begin{array}{l}
x_{2}^{c}(k+1)=f_{2}\left(x_{1}^{\prime}(k), x_{2}^{c}(k), u(k)\right) \\
u(k)=c_{2}\left(x_{1}^{\prime}(k), x_{2}^{c}(k), v_{\mathrm{des}}(k)\right) \\
v(k)=g\left(x_{2}(k)\right) \\
\tilde{v}(k)=v(k)-v_{\mathrm{des}}^{f}(k) \\
x_{f}(k+1)=f_{3}\left(x_{f}(k), v_{\mathrm{des}}(k)\right) \\
v_{\mathrm{des}}^{f}(k)=g_{f}\left(x_{f}(k)\right)
\end{array}\right.
\end{aligned}
$$

where $\Sigma_{1}$ and $\Sigma_{2}$ denote the outer loop and inner loop systems with their corresponding controllers, $c_{1}\left(x_{1}^{c}(k), r(k)\right)$ and $c_{2}\left(x_{1}^{\prime}(k), x_{2}^{c}(k), v_{\text {des }}(k)\right)$, respectively. The states $x_{f}$ represent those of the inner loop reference model, an identical copy of which is embedded in each subsystem $\left(\Sigma_{1}\right.$ and $\left.\Sigma_{2}\right)$. This reflects the design principle that, even if the inner and outer loop designs are carried out in parallel, knowledge of the reference model is common to both designers. For analysis purposes, it is assumed that the reference model states are initialized to the same values in both copies. In (2) and (3), the states, $x_{1}^{c}$ and $x_{2}^{c}$ are those of the closed-loop system, thereby also containing controller states.

Given this design process, it is important to characterize what constitutes a "desirable and realistic" reference model. While the specific choice of reference model will depend on the application at hand, the reference model should at a minimum have a relative degree that is greater than or equal to the relative degree from $u$ to $v$, where the relative degree is defined as follows [17].

Definition 3.1: $\Sigma_{2}$ has (strict) relative degree $\rho$ from $u$ to $v$, which is well-defined everywhere, if the following hold:

1) $\forall i<\rho, \forall x(k) \in \mathbb{R}^{n},(\partial v(k+i)) / \partial u(k)=0_{1 \times q}$; 2) $\forall x(k) \in \mathbb{R}^{n}, \forall u(k) \in \mathbb{R}^{q},(\partial v(k+\rho)) / \partial u(k) \neq 0_{1 \times q}$. 
It should be noted that for an overactuated system (like the thermal management system), $\partial v(k+i) / \partial u(k)$ is a vector, and only one element of that vector needs to be nonzero for the vector to be nonzero (which, in turn, implies that the relative degree of the reference model corresponds to the lowest relative degree among all of the actuators). Without imposing this relative degree requirement on the reference model, it becomes impossible for the inner loop to match the behavior that is specified by the reference model under a causal controller.

\section{B. MPCA and Predictor Structure}

The purpose of the inner loop control allocation is to generate closed-loop behavior for $\Sigma_{2}$ that closely matches the inner loop reference model in the presence of saturation constraints and different dynamic actuator authorities. In order to do this, the inner loop controller is formulated as a standard model predictive control problem, as described in detail in [18]. MPCA considers an optimization in which the following cost function is minimized over a receding horizon:

$$
\begin{aligned}
J\left(x_{2}(k), \mathbf{u}(k)\right) & =\sum_{i=k}^{k+N-1} l\left(\hat{x}_{2}(i \mid k), \hat{v}_{\mathrm{des}}^{f}(i \mid k), u(i \mid k)\right), \\
\mathbf{u}(k) & =\left[\begin{array}{lll}
u(k \mid k) & \ldots & u(k+N-1 \mid k)
\end{array}\right]^{T}
\end{aligned}
$$

subject to the constraints

$$
u(i \mid k) \in U=\left\{u: u_{\min , j} \leq u_{j} \leq u_{\max , j}, 1 \leq j \leq q\right\}
$$

where

$$
\begin{aligned}
l\left(\hat{x}_{2}(i \mid k), \hat{v}_{\text {des }}^{f}(i \mid k), u(i \mid k)\right)= & \left(\hat{v}(i \mid k)-\hat{v}_{\text {des }}^{f}(i \mid k)\right)^{2} \\
& +P\left(\hat{x}_{2}(i \mid k), u(i \mid k)\right)
\end{aligned}
$$

and $N$ is the length of the prediction horizon. The first element of the optimal control sequence is implemented, and the optimization is repeated at step $k+1$. Thus, the MPCA control law is

$$
u(k)=u^{o}(k \mid k)
$$

where

$$
\begin{aligned}
\mathbf{u}^{o}(k) & \triangleq \arg \min J\left(x_{2}(k), \mathbf{u}(k)\right) \\
& =\left[\begin{array}{lll}
u^{o}(k \mid k) & \ldots & u^{o}(k+N-1 \mid k)
\end{array}\right]^{T} .
\end{aligned}
$$

The notation $\hat{v}$ (where $v$ could be replaced with other variables) denotes a prediction, rather than the actual value, and the notation $(i \mid k)$ denotes the value at step $i$, where the prediction, or optimization in the case of $u(i \mid k)$, is being made at step $k$.

The first term in the incremental cost, $l\left(\hat{x}_{2}(i \mid k), \hat{v}_{\mathrm{des}}^{f}(i \mid k), u(i \mid k)\right), \quad$ penalizes deviation from the inner loop reference model, whereas the second can be used to shape the response of the closed-loop system. The optimization requires a prediction of $\hat{v}_{\mathrm{des}}^{f}(i \mid k), i=0 \ldots N-1$, which is accomplished through an outer loop predictor, specified by

$$
\begin{aligned}
\hat{x}_{1}(i+1 \mid k) & =f_{1}\left(\hat{x}_{1}(i \mid k), \hat{v}_{\mathrm{des}}^{f}(i \mid k), \hat{\tilde{v}}(i \mid k)\right) \\
\hat{v}_{\mathrm{des}}(i \mid k) & =c_{1}\left(\hat{x}_{1}(i \mid k), \hat{r}(i \mid k)\right)
\end{aligned}
$$

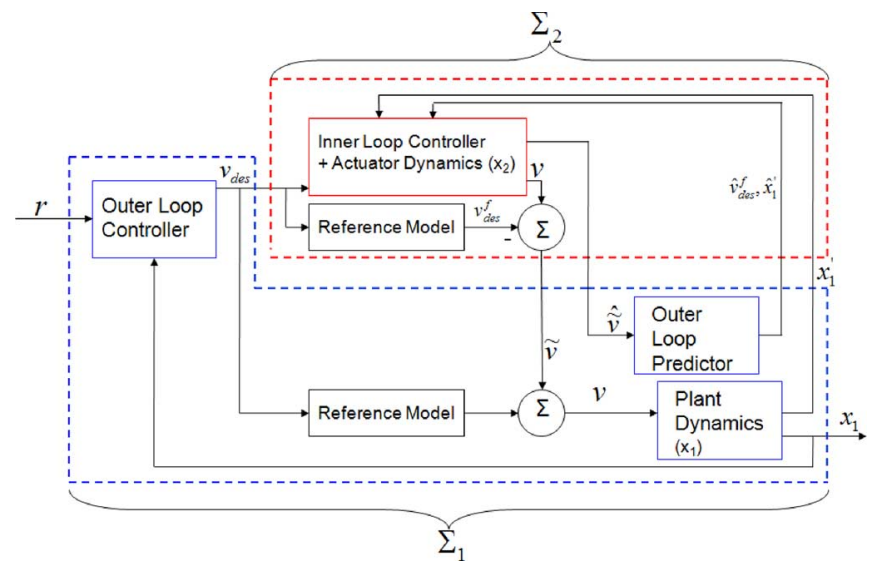

Fig. 8. Diagram of the system (1) recast for the reference model based design framework with inner loop MPCA.

$$
\begin{aligned}
\hat{x}_{1}^{\prime}(i \mid k) & =d\left(\hat{x}_{1}(i \mid k)\right) \\
\hat{x}_{f}(i+1 \mid k) & =f_{3}\left(\hat{x}_{f}(i \mid k), \hat{v}_{\mathrm{des}}(i \mid k)\right) \\
\hat{v}_{\mathrm{des}}^{f}(i \mid k) & =g_{f}\left(\hat{x}_{f}(i \mid k)\right) .
\end{aligned}
$$

Note that MPCA by its nature automatically derives a prediction of the states over the receding horizon, i.e., $\hat{\hat{v}}(i \mid k), i=$ $0, \ldots, N$, so there is no need for an additional inner loop predictor. The incorporation of an outer loop predictor results in a modified system diagram, given by Fig. 8, which preserves the structure of Fig. 7 but incorporates prediction of $v_{\text {des }}$ over the full horizon. The design of this predictor is within the design scope of the outer loop (hence, it lies within the dashed lines belonging to $\Sigma_{1}$ ), because it requires knowledge of the plant and outer loop controller dynamics.

\section{APPlication OF MPCA TO THERMAL MANAGEMENT-CONTROL DESIGN}

Referring to the terminology and nomenclature introduced in [13] and summarized in Section II, in Table I, the thermal management system is cast in the form specified by (1). Taking the virtual control input as the mixing valve outlet temperature, $T_{\text {out,mv }}$, the plant states $\left(x_{1}\right)$ include the engine outlet temperature, $T_{\text {out,eng }}$, and the engine block temperatures, $T_{\text {eng1,2 }}$. The actuator states $\left(x_{2}\right)$ include the mixing valve outlet temperature, $T_{\text {out,mv }}$, the heat exchanger outlet temperature, $T_{\text {out,hex }}$, the heater outlet temperature, $T_{\text {out,ht }}$, the heater coil temperature, $T_{\mathrm{ht}}$, and the cooling water temperature at the outlet of the heat exchanger, $T_{\text {out }, \mathrm{cw}}$.

The controller design is divided into three components, namely the design of the inner loop reference model, the design of the outer loop controller, and the design of the MPCA optimization. In this application, achieving a settling time of approximately $60 \mathrm{~s}$ (or as quickly as possible in low speed and load conditions when $60 \mathrm{~s}$ is impossible due to limited heater power), with minimal or no overshoot, is of paramount interest. Following the design of the reference model and outer loop controller, the performance of MPCA (or any inner loop controller) can be evaluated by comparing the actual system response with the ideal response that is achieved when the reference model is matched exactly. 


\section{A. Inner Loop Reference Model Design}

Since the reference model represents a design target from the inner loop perspective and a design assumption on the part of the outer loop, the following properties describe an ideal reference model.

- If the model of the actuator dynamics is perfect, then the reference model can be matched (i.e., the inner closed loop performance can be made equal to that of the reference model, which will lead $\tilde{v}$ to asymptotically approach zero).

- The closed-loop system is robust to uncertainties with the chosen reference model.

It can be easily verified that the first property requires that the reference model has relative degree that is equal to or greater than the relative degree of the actuator dynamics, from $u$ to $v$. The second property prohibits the use of overly aggressive reference models that pursue too high of an inner closed loop bandwidth to ensure robust performance in the presence of uncertainty. Additionally, since the introduction of a strictly causal reference model introduces phase lag, a slow reference model will typically lead to diminished phase margins, and, consequently, deteriorated stability robustness. While the first property can be verified at the outset of the design, the second requires some engineering judgment, as the precise analysis of the effects of uncertainty depends upon the actual outer and inner loop controllers that are used.

Observing that the actuator dynamics have relative degree 1, the simplest relative degree 1 reference model is chosen, namely a first-order filter with time constant $\tau_{r}$

$$
F(s)=\frac{1}{\tau_{r} s+1} .
$$

For this reference model, the key is choosing $\tau_{r}$ such that the reference model can be matched reasonably closely in the presence of uncertainty and that the reference model does not introduce excessive phase lag at frequency ranges of interest.

\section{B. Outer Loop Control Design}

The outer loop control design is conducted by replacing the closed inner loop dynamics with $F$ and proceeding using linear system design and analysis tools. Referring back to the outer loop design framework shown in Fig. 5, it is clear that the objective of the outer loop controller is to achieve setpoint tracking of $r$ and disturbance rejection of $\tilde{v}$. In light of this fact, verification of the outer loop controller performance can be obtained through the following three means:

1) examining Bode plots from $r$ and $\tilde{v}$ to $y$ to verify outer loop tracking and disturbance $(\tilde{v})$ rejection performance;

2) examining the Bode plot from $e \triangleq r-y$ to $y$, to ascertain the phase margin;

3) examining simulation results that are obtained when the inner closed loop is replaced with the reference model, which gives a time domain interpretation of the content examined in (1).

Linear design tools (Root loci and Bode plots), as well as simulations where the inner loop is replaced with $F(s)$, indicate that a PI controller, given by

$$
C(s)=\frac{2 s+0.05}{s} \quad C(z)=\frac{2 z-1.95}{z-1}
$$

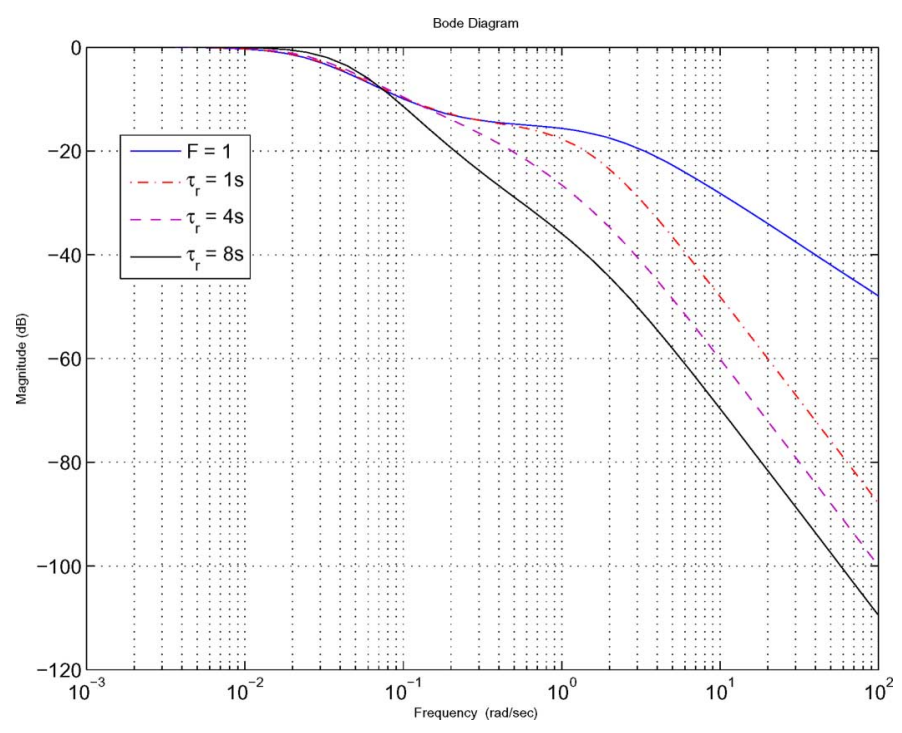

Fig. 9. Closed-loop dynamics from $T_{\text {out,eng }}^{\text {des }}(r)$ to $T_{\text {out,eng }}(y)$ when the inner loop reference model is matched exactly.

(where the implementation sampling time is again $1 \mathrm{~s}$ ) yields desirable results, which are shown in Figs. 9-11. Figs. 9 and 10 verify system performance under the selected reference model, comparing different reference model time constants, namely, $\tau_{r}=1, \tau_{r}=4 \mathrm{~s}$, and $\tau_{r}=8 \mathrm{~s}$, in addition to the reference model $F=1$. At this point, all choices of reference models appear to work well with the chosen outer loop controller (8). However, although $F=1$ seems to yield satisfactory performance, it is impossible to match with relative degree 1 actuator dynamics. A reference model with $\tau_{r}=8 \mathrm{~s}$ on the other hand, can likely be matched closely, but yields very small phase margins, as exhibited in Fig. 11. Thus, simulation and experimental results will consequently focus on reference models with $\tau_{r}=$ $1 \mathrm{~s}$ and $\tau_{r}=4 \mathrm{~s}$.

\section{MPCA Optimization}

Given a well-designed reference model and outer loop controller, the objective of the inner loop MPCA is to leverage the capabilities of multiple actuators (two, in this case) to drive $v$ to the output of the reference model, $v_{\mathrm{des}}^{f}$. To set up the MPCA optimization, the cost function, horizon length, and optimization algorithm must be selected.

For this application, the inner loop sampling time is taken as $1 \mathrm{~s}$ (same as the outer loop), with the cost function specified by (4) with $P\left(x_{2}, u\right)=0$. The horizon length for the MPCA optimization is chosen to be $30 \mathrm{~s}$ (30 steps), which is reflective of the time constants associated with the inner loop reference model as well as the heater and heat exchanger.

The online MPCA optimization used in this paper relies on the computation of the sensitivity function, as in [19], which captures the sensitivity of the cost function to the control inputs. This method has been shown in [19] to be more efficient than DP or SQP methods (for a particular large-scale ship application) and is capable of handling saturation constraints. The sensitivity function provides a search direction along which a one-dimensional optimization is performed to determine the minimum value of the cost function. The process is carried out iteratively 


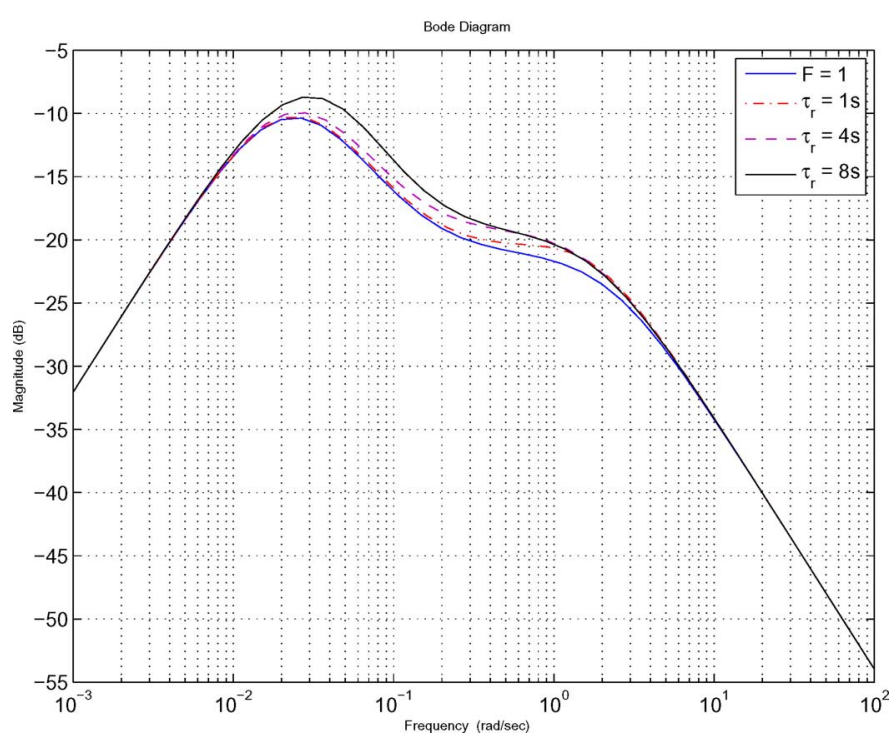

Fig. 10. Closed-loop dynamics from $T_{\text {out }, \mathrm{mv}}-T_{\text {out }, \text { mv }}^{\text {des, }}(\tilde{v})$ to $T_{\text {out,eng }}(y)$ when the inner loop reference model is matched exactly.

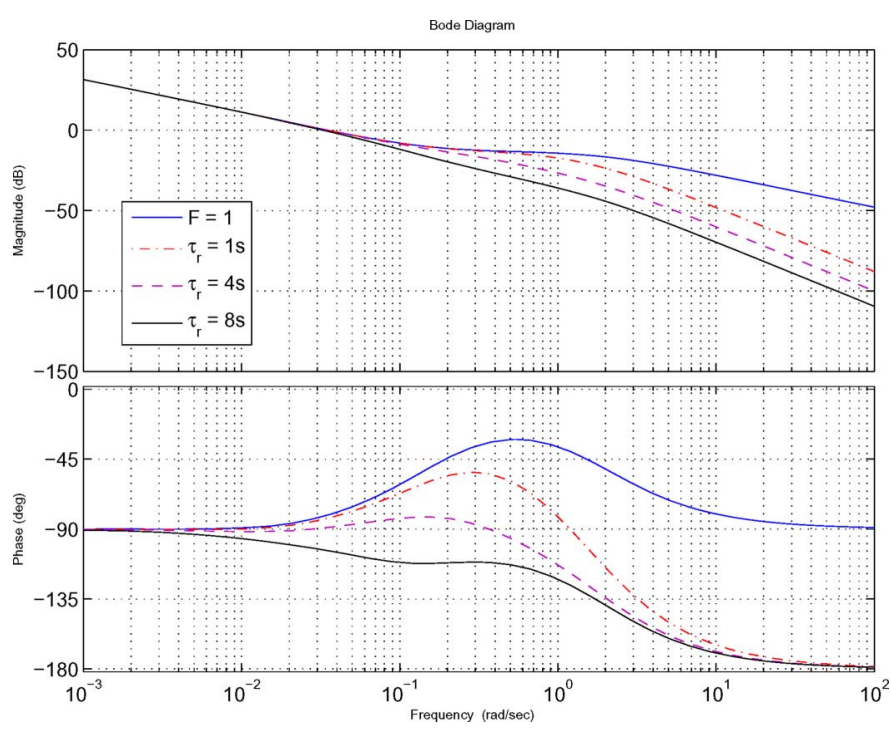

Fig. 11. Open loop transfer function - From $T_{\text {out,eng }}^{\text {des }}-T_{\text {out,eng }}(r-y)$ to $T_{\text {out,eng }}(y)$.

until the optimal cost converges, based on a prescribed convergence criterion, or a maximum number of iterations has been reached. Readers are referred to [19] for the details of implementation.

\section{APPLICATION OF MPCA TO THERMAL MANAGEMENT-REAL-TIME SimUlation RESUltS}

Prior to implementing the proposed controller on the thermal management system, simulations were performed on the University of Michigan Real Time and Adaptive Control Engineering RACE Lab's OpalRT real time simulator. This served two purposes, namely the following:

1) simulations allowed us to tune controller parameters and verify anticipated controller performance prior to implementing on the physical thermal management system,
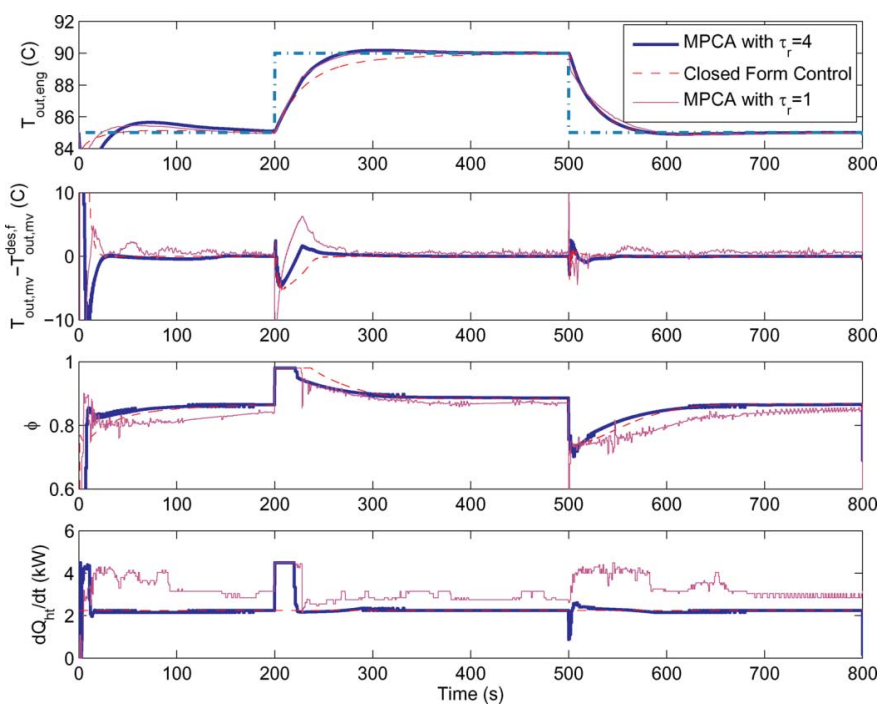

Fig. 12. Simulated system behavior.

thereby reducing the time required for experimental validation;

2) the real time feature allowed us to verify the computational feasibility of the chosen MPCA optimization algorithm.

In order to provide a benchmark control strategy against which to compare the MPCA strategy, MPCA is compared against a closed-form controller, given by

$$
\begin{aligned}
& \phi(k)=\operatorname{sat}\left(\frac { 1 } { T _ { \text { out } , \text { ht } } - T _ { \text { out } , \text { hex } } } \left(( 1 - \frac { \tau _ { t } } { \tau _ { r } } ) \left(T_{\text {out }, \text { mv }}^{\text {des }}\right.\right.\right. \\
& \left.\left.\left.-T_{\text {out }, \mathrm{mv}}\right)+T_{\text {out }, \mathrm{mv}}^{\mathrm{des}}-T_{\text {out,hex }}\right)\right) \\
& \dot{Q}_{h t}(k)=2.25
\end{aligned}
$$

which results in closed inner loop performance that matches the reference model exactly when saturation is not active. Here, the heater is held at a constant power (one that is desirable for the engine speed and load conditions), which has been employed in previous thermal management strategies due to the difficulty in effectively incorporating the heater into the controller [13].

Real-time simulation results are shown for the nominal system model in Fig. 12. These simulations are based on a test condition with an engine speed of $2000 \mathrm{r} / \mathrm{min}$ and load of 75 $\mathrm{N} \cdot \mathrm{m}$. Results demonstrate that reference model-based MPCA uses both actuators effectively in order to provide more accurate tracking than under the benchmark (closed form) controller. In the case when an aggressive reference model with $\tau_{r}=1 \mathrm{~s}$ is used, MPCA produces erratic control inputs, although setpoint tracking remains unharmed.

In order to simulate the effect of uncertain actuator dynamics on the system performance, the nominal actuator dynamics are augmented with flow rate dynamics, where flow rate does not change immediately upon actuation of the mixing valve, but rather based on a time constant of $\tau_{\text {flow }}=2 \mathrm{~s}$, which leads to the augmented actuator dynamics given by

$$
\dot{\phi}=\frac{1}{\tau_{\text {flow }}}\left(-\phi+u_{\mathrm{mv}}\right) \text {. }
$$



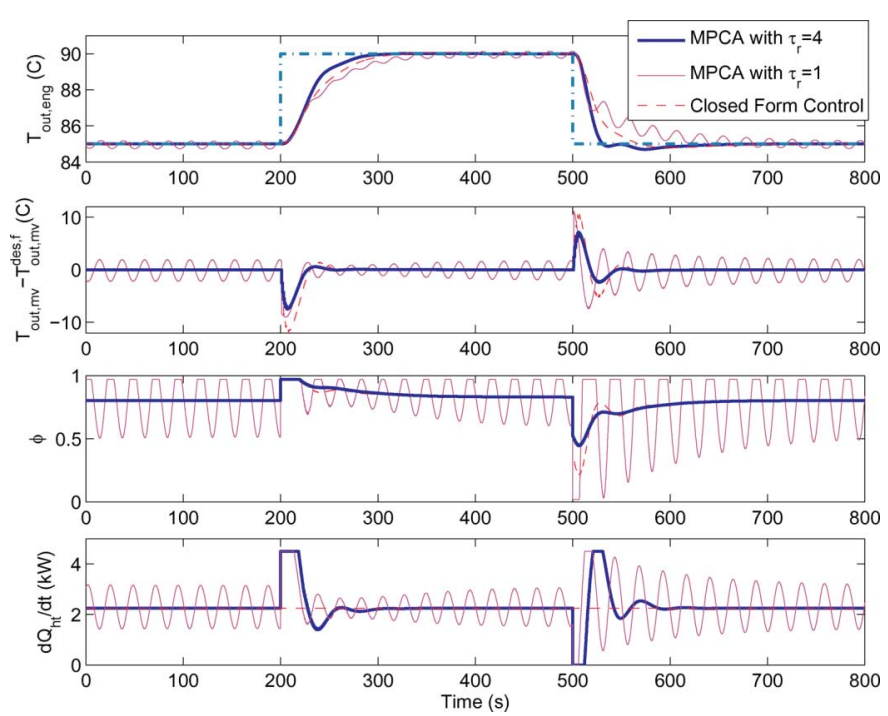

Fig. 13. Simulated system behavior in the presence of unmodeled actuator dynamics.

Additionally, $T_{\mathrm{in}, \mathrm{cw}}$ (the cooling water inlet temperature) is lowered from 20 to $30 \mathrm{C}$ to introduce an unmodeled bias in the required mixing valve position. The simulation results with this uncertainty are shown in Fig. 13. Here, it is seen that the system responds similarly to the nominal system when we take $\tau_{r}=4$, but with a more aggressive reference model time constant of $\tau_{r}=1 \mathrm{~s}$, significant oscillations are introduced. These oscillations are more pronounced at the innermost levels of the system (actuators and virtual control) but are also present in the setpoint tracking response. This aggressive reference model $\left(\tau_{r}=1 \mathrm{~s}\right)$ will be reexamined in the experimental results to show that reference model selection is indeed an important concern in modular control with MPCA.

\section{APPLiCATION OF MPCA TO THERMAL MANAGEMENT-EXPERIMENTAL VALIDATION}

In the experimental setup, depicted in Fig. 14, the controller and hardware interface are designed using MATLAB Real Time Workshop and XPC Target. The configuration relies on a host PC where all of the control design takes place, and a target PC, which executes compiled $\mathrm{C}$ code and transmits/receives signals through two rapid prototyping boards. One board, the Measurement Computing PCI-DAS-TC board, handles all of the thermocouple measurements (with locations depicted in Fig. 1), whereas the other board, the Quanser Q4, handles the analog inputs (flow rate measurements, with locations depicted in Fig. 1) and outputs (mixing valve and heater commands).

Experimental results were acquired for identical test cases as those used in simulation (engine speed of $2000 \mathrm{r} / \mathrm{min}$ and load of $75 \mathrm{~N} \cdot \mathrm{m}$ ) and are provided in Fig. 15. Like the simulation results, the experimental results show that the use of MPCA allows the system to reach the setpoint more quickly through effective use of both actuators.

It can be seen, however, that when $\tau_{r}$, is decreased to $1 \mathrm{~s}$, the experimental response exhibits significant oscillations that are

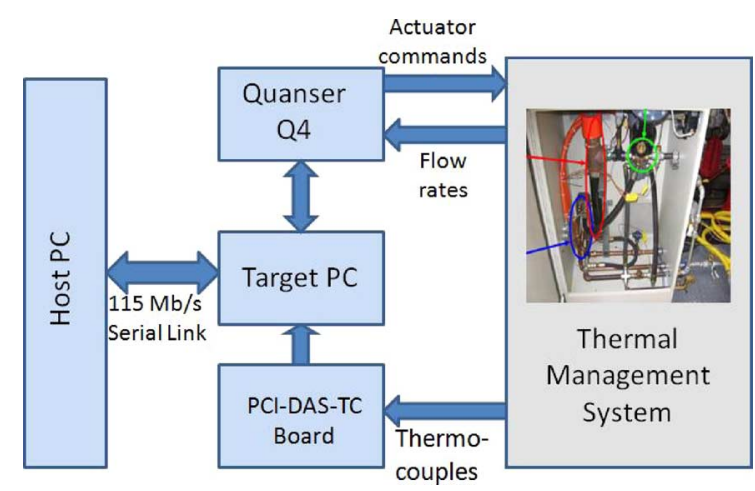

Fig. 14. Thermal management rapid prototyping configuration.
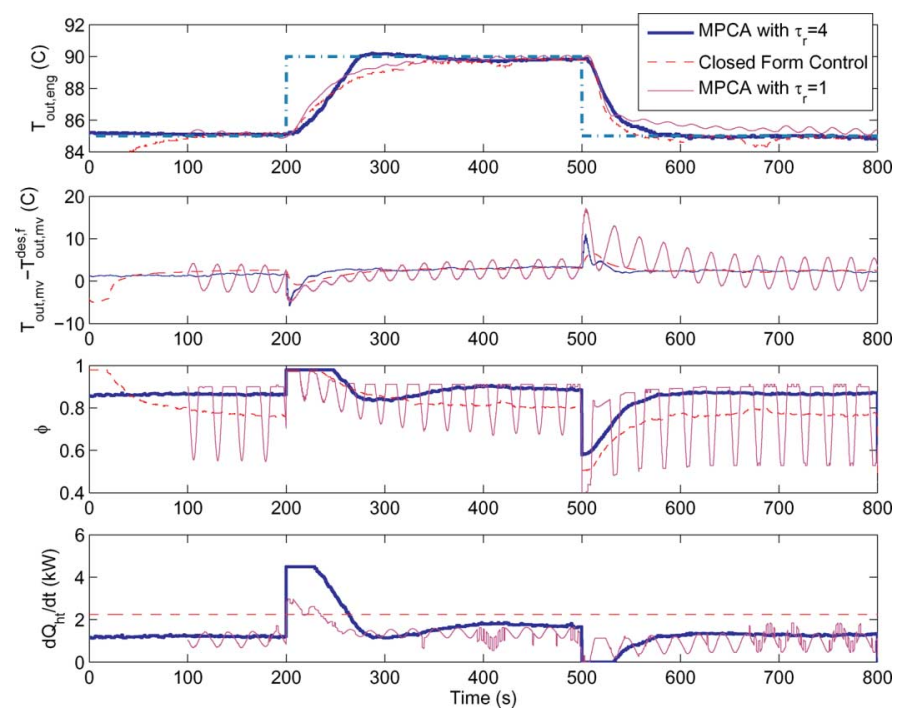

Fig. 15. Experimental results.

not predicted in simulation results. With such an aggressive reference model, the closed-loop performance is significantly affected by uncertainties in the model of the fluid conditioning system (the actuator dynamics). This underscores the importance of choosing the reference model to have an appropriate bandwidth for the system at hand and helps to show the benefit of incorporating the reference model as a design freedom in the application of MPCA to systems with nontrivial actuator dynamics.

Certain characteristics are present in the experimental results that are not present in simulations, which warrant two remarks.

Remark 6.1: The slight offset between $v$ and $v_{\text {des }}^{f}$ throughout Fig. 15, which is even present at steady state, is due to the fact that there is no integrator for the inner loop, and the inner loop model is not perfect. In this system, the integrator present in the outer loop controller is sufficient for steady-state tracking of the desired engine outlet temperature.

Remark 6.2: The rate at which the temperature rises upon full (saturated) application of the mixing valve is dependent on the rate of heat rejection into the system, both through the heater and the engine. While the speed and load are regulated closely by the dynamometer, they do possess some variation, and environmental conditions do vary from test to test. Consequently, results often do show slightly different transient responses even 
TABLE II

DYNAMIC MODEL

\begin{tabular}{|c|c|}
\hline Subsystem & Equations \\
\hline Mixing Valve & $\dot{T}_{\text {out }, m v}=\frac{1}{\tau_{t}}\left(\phi T_{\text {out }, h t}+(1-\phi) T_{\text {out }, \text { hex }}\right)$ \\
\hline Heat Exchanger & 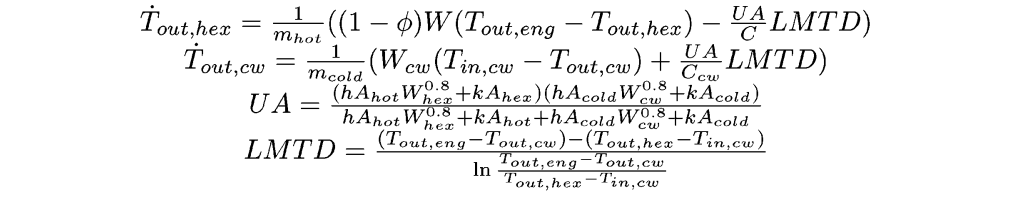 \\
\hline Heater & $\begin{aligned} \dot{T}_{\text {out }, h t}= & \frac{1}{m_{\text {fluid }}}\left(\phi W\left(T_{\text {out }, \text { eng }}-T_{\text {out }, h t}\right)+\left(\frac{h A_{h t} W_{h t}^{8}+k A_{h t}}{C}\right)\left(T_{h t}-T_{\text {out }, h t}\right)\right) \\
& \dot{T}_{h t}=\frac{1}{m C_{h t}}\left(\dot{Q}_{h t}-\left(h A_{h t} W_{h t}^{8}+k A_{h t}\right)\left(T_{h t}-T_{\text {out }, h t}\right)\right)\end{aligned}$ \\
\hline Engine & $\begin{array}{c}\dot{T}_{\text {out }, \text { eng }}^{c}=\frac{1}{m^{c}}\left(W^{c}\left(T_{\text {out }, \text { eng }}^{c}-T_{\text {out }, m v}^{c}\right)+\left(\frac{h A^{c}\left(W^{c}\right)^{8}+k A^{c}}{C^{c}}\right)\left(T_{\text {eng } 1}-T_{\text {out }, \text { eng }}^{c}\right)\right) \\
\dot{T}_{\text {out }, \text { eng }}^{o}=\frac{1}{m^{o}}\left(W^{o}\left(T_{\text {out }, \text { eng }}^{o}-T_{\text {out }, m v}^{o}\right)+\left(\frac{h A^{o}\left(W^{o}\right)^{8}+k A^{o}}{C^{o}}\right)\left(T_{\text {eng } 2}-T_{\text {out }, \text { eng }}^{o}\right)\right) \\
\dot{T}_{\text {eng } 1}=\frac{1}{m C_{\text {eng } 1}}\left(\dot{Q}_{1}+k A_{c p}\left(T_{\text {eng } 2}-T_{\text {eng } 1}\right)-\left(h A^{c}\left(W^{c}\right)^{8}+k A^{c}\right)\left(T_{\text {eng } 1}-T_{\text {out }, \text { eng }}^{c}\right)\right) \\
\dot{T}_{\text {eng } 2}=\frac{1}{m C_{\text {eng } 2}}\left(\dot{Q}_{2}+k A_{c p}\left(T_{\text {eng } 1}-T_{\text {eng } 2}\right)-\left(h A^{o}\left(W^{o}\right)^{8}+k A^{o}\right)\left(T_{\text {eng } 2}-T_{\text {out }, \text { eng }}^{o}\right)\right.\end{array}$ \\
\hline
\end{tabular}
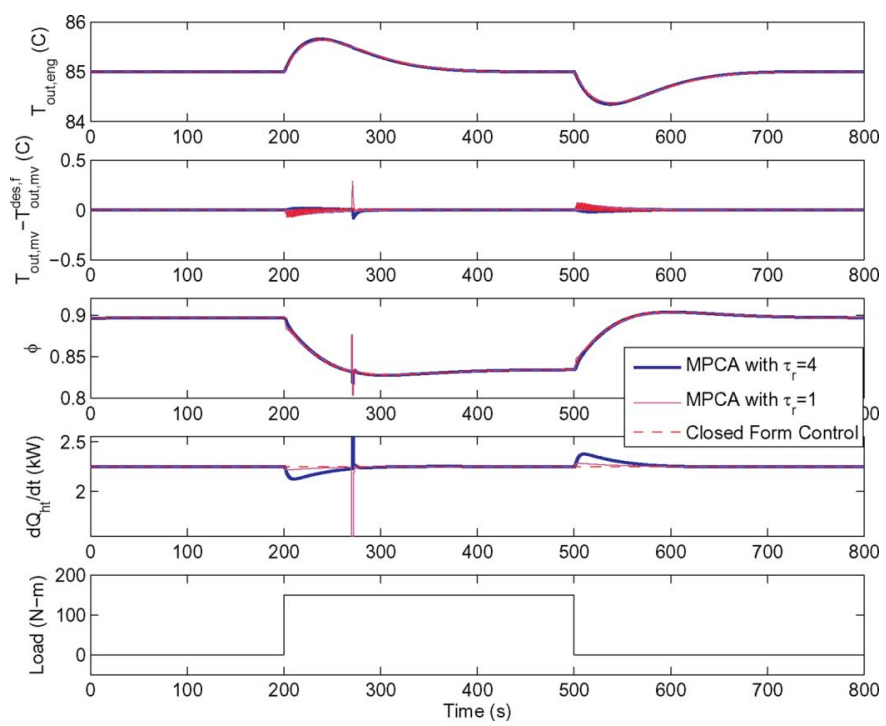

Fig. 16. Simulated disturbance rejection behavior.

when the valve is at full saturation (for example, in Fig. 15, the trace acquired with $\tau_{r}$ exhibits slower initial response in spite of the valve being initially at saturation in all three cases).

\section{CONCLUSION AND FUTURE WORK}

This paper proposes a reference model based version of MPCA that is applied on a thermal management system. This paper has shown the entire design process and demonstrated through real-time simulation and experimental results that MPCA does an effective job of blending the effects of two different actuators in order to improve control system performance. It has also demonstrated the importance of appropriate reference model selection in shaping the response of the overall system. Future work will involve the development of deeper theoretical insights with regard to the stability and guaranteed levels of performance with the reference model-based approach.
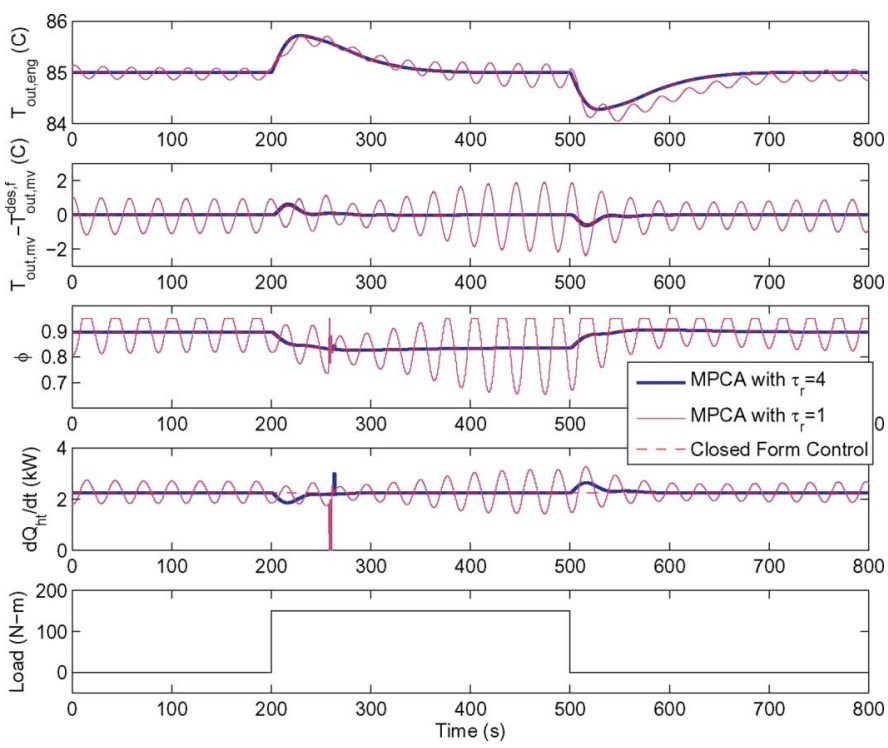

Fig. 17. Simulated disturbance rejection behavior in the presence of unmodeled actuator dynamics.

\section{APPENDIX \\ Disturbance ReJeCtion PERFORMANCE}

While the main focus of this paper was on setpoint tracking, the use of reference model-based MPCA can also be extended to disturbance rejection. In fact, many routine engine thermal management applications involve the adjustment of speed and load while striving to maintain a constant engine outlet temperature. In this appendix, simulation results are presented to illustrate the effectiveness of the proposed control design in a disturbance rejection framework.

Figs. 16 and 17 consider a load disturbance, from 0 to 150 $\mathrm{N} \cdot \mathrm{m}$ at $200 \mathrm{~s}$, then back to $0 \mathrm{~N} \cdot \mathrm{m}$ at $500 \mathrm{~s}$. This load change has the effect of altering $\dot{Q}_{1,2}$ (the heat rejection from the engine). Fig. 16 refers to the nominal case (a perfect model), whereas Fig. 17 considers the same uncertainty that was considered in the setpoint tracking simulations with uncertainty. Fig. 16, shows that good disturbance rejection performance is obtained with and without MPCA. The nearly equal performance with and 
without MPCA arises due to the fact that the disturbance does not trigger saturation in the mixing valve; thus, under the ideal situation where all model parameters are known, both the closed form controller and MPCA are capable of obtaining perfect reference model matching. Fig. 17 shows the familiar effect of an overly aggressive reference model in the presence of uncertainties, wherein large oscillations are induced.

\section{ACKNOWLEDGMENT}

The authors would like to thank A. Vickery of IAV Automotive and B. Moore of A and D Technology for staying in the test cell after normal hours to help to run experimental validation tests.

\section{REFERENCES}

[1] T. Johansen, T. Fossen, and S. Berge, "Constrained nonlinear control allocation with singularity avoidance using sequential quadratic programming," IEEE Trans. Control Syst. Technol., vol. 12, no. 1, pp. 211-216, Jan. 2004.

[2] O. J. Sordalen, "Optimal thrust allocation for marine vessels," Control Eng. Pract., vol. 5, no. 9, pp. 1223-1231, 1997.

[3] O. Harkegard, "Resolving actuator redundancy - control allocation vs. linear quadratic control," presented at the Euro. Control Conf., Cambridge, U.K., 2003.

[4] M. Bodson, "Evaluation of optimization methods for control allocation," J. Guid., Control, Dyn., vol. 25, no. 4, pp. 703-711, 2002.

[5] W. Durham, "Attainable moments for the contstrained control allocation problem," J. Guid., Control, Dyn., vol. 17, no. 6, pp. 1371-1373, 1994.

[6] J. Tjonnas and T. Johansen, "Optimizing adaptive control allocation with actuator dynamics," presented at the IEEE Conf. Dec. Control, New Orleans, LA, 2007.

[7] Y. Luo, A. Serrani, S. Yurkovich, D. Doman, and M. Oppenheimer, "Model predictive dynamic control allocation with actuator dynamics," presented at the Amer. Control Conf., Boston, MA, 2004.

[8] Y. Luo, A. Serrani, S. Yurkovich, D. Doman, and M. Oppenheimer, "Dynamic control allocation with asymptotic tracking of time-varying control input commands," presented at the Amer. Control Conf., Portland, OR, 2005.

[9] Y. Luo, A. Serrani, S. Yurkovich, M. Oppenheimer, and D. Doman, "Model predictive dynamic control allocation scheme for reentry vehicles," J. Guid., Control, Dyn., vol. 30, no. 1, pp. 100-113, 2007.

[10] C. Vermillion, J. Sun, and K. Butts, "Model predictive control allocation - stability and performance," presented at the IEEE Conf. Dec. Control, New Orleans, LA, 2007.

[11] R. Scattolini and P. Colaneri, "Hierarchical model predictive control," presented at the IEEE Conf. Dec. Control, New Orleans, LA, 2007.

[12] C. Vermillion, J. Sun, and K. Butts, "Performance enhancement of modular control systems using $\mu$ synthesis," presented at the IEEE Conf. Dec. Control, Cancun, Mexico, 2008.

[13] C. Vermillion, J. Sun, and K. Butts, "Modeling, control design, and experimental validation for an overactuated engine thermal management system for engine dynamometer applications," IEEE Trans. Control Syst. Technol., vol. 17, no. 3, pp. 540-551, May 2009.

[14] C. Vermillion, "Optimal modular control of overactuated systems Theory and applications," Ph.D. dissertation, Dept. Elect. Eng., Univ. Michigan, Ann Arbor, 2009.
[15] R. Sonntag and C. Borgnakke, Fundamentals of Thermodynamics. New York: Wiley, 2002.

[16] P. Ioannou and J. Sun, Robust Adaptive Control. Englewood Cliffs, NJ: Prentice-Hall, 1996.

[17] D. Liberzon, A. Morse, and E. Sontag, "Output-input stability and minimum-phase nonlinear systems," IEEE Trans. Autom. Control, vol. 47, no. 3, pp. 422-436, Mar. 2002.

[18] D. Mayne, J. Rawlings, C. Rao, and P. Scokaert, "Constrained model predictive control: Stability and optimality," Automatica, vol. 36, no. 6, pp. 789-814, 2000 .

[19] G. Seenumani, J. Sun, and H. Peng, "A numerically efficient iterative procedure for hybrid power system optimization using sensitivity functions," presented at the Amer. Control Conf., New York, 2007.

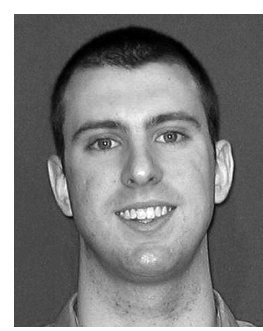

Chris Vermillion (M’09) received the B.S.E. degree in aerospace engineering and mechanical engineering, and the M.S. and Ph.D. degrees in electrical engineering, from the University of Michigan, Ann Arbor, in 2004, 2005, and 2009, respectively.

$\mathrm{He}$ is currently with Toyota Technical Center, Ann Arbor, MI, where he works on advanced powertrain research and development. His research interests include advanced powertrain control, modular control strategies, and optimal control. He is currently investigating the use of model predictive control as an alternative to legacy powertrain control strategies.

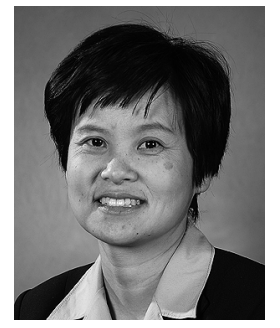

Jing Sun (F'04) received the Ph.D. degree from the University of Southern California, Los Angeles, in 1989, and the B.S. and M.S. degrees from University of Science and Technology of China, Hefei, China, in 1982 and 1984, respectively.

From 1989 to 1993, she was an Assistant Professor with the Department of Electrical and Computer Engineering, Wayne State University. She joined Ford Research Laboratory in 1993 where she worked in the Powertrain Control Systems Department. After spending almost 10 years in industry, she came back to academia and joined the faculty of the College of Engineering, University of Michigan, Ann Arbor, in 2003, where she is currently a Professor with the Department of Naval Architecture and Marine Engineering and Department of Electrical Engineering and Computer Science. Her research interests include system and control theory and its applications to marine and automotive propulsion systems. She holds over 30 U.S. patents and has coauthored a textbook on robust adaptive control.

Dr. Sun was a one of three recipients of the 2003 IEEE Control System Technology Award.

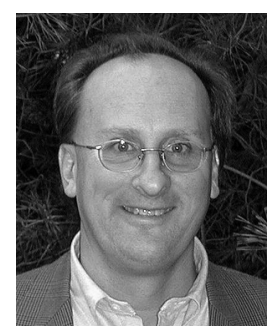

Ken Butts (M'10) received the B.E. degree in electrical engineering from General Motors Institute (now Kettering University), Flint, MI, the M.S. degree in electrical engineering from the University of Illinois, Urbana-Champaign, and the Ph.D. degree in electrical engineering systems from the University of Michigan, Ann Arbor.

$\mathrm{He}$ is an Executive Engineer with the Powertrain and Chassis Division, Toyota Motor Engineering and Manufacturing North America, Ann Arbor, MI, where he is investigating methods to improve engine control development productivity. 